\title{
Integrating Behavioral and Physical Health Care in the Real World: Early Lessons from Advancing Care Together
}

\author{
Melinda Davis, PhD, Bijal A. Balasubramanian, MBBS, PhD, Elaine Waller, BA, \\ Benjamin F. Miller, PsyD, Larry A. Green, MD, and Deborah J. Cohen, PhD
}

Background: More than 20 years ago the Institute of Medicine advocated for integration of physical and behavioral health care. Today, practices are integrating care in response to recent policy initiatives. However, few studies describe how integration is accomplished in real-world practices without the financial or research support available for most randomized controlled trials.

Methods: To study how practices integrate care, we are conducting a cross-case comparative, mixedmethods study of 11 practices participating in Advancing Care Together (ACT). Using a grounded theory approach, we analyzed multiple sources of data (eg, documents, practice surveys, field notes from observation visits, semistructured interviews, online diaries) collected from each ACT innovator.

Results: Integration requires making changes in organization and interpersonal relationships. During early integration efforts, challenges related to workflow and access, leadership and culture change, and tracking and using data to evaluate patient- and practice-level improvement emerged for ACT innovators. We describe the strategies innovators are developing to address these challenges.

Conclusion: Integrating care is a fundamental and difficult change for practices and health care professionals. Research identifying common challenges that manifest in early efforts can help others attempting integration and inform state, local, and federal policies aimed at achieving wide-spread implementation. (J Am Board Fam Med 2013;26:588-602.)

Keywords: Implementation Research, Integrated Care, Mental Health, Mixed Methods, Primary Health Care

It has been almost 20 years since the Institute of Medicine (IOM) declared primary care and behavioral health to be inseparable. ${ }^{1}$ In the interval, the Agency for Health care Research and Quality, ${ }^{2,3}$ the $\mathrm{IOM}^{4}{ }^{4}$ and other state and federal agencies have advocated for integrating delivery of physical and be-

This article was externally peer reviewed.

Submitted 14 January 2013; revised 30 April 2013; accepted 13 May 2013.

From the Department of Family Medicine, Oregon Health \& Science University, Portland (MD, EW, DJC); University of Texas Health Science Center, Houston, School of Medicine, Dallas (BAB); the Office of Integrated Healthcare Research and Policy (BFM) and the Department of Family Medicine (BFM, LAG), University of Colorado, Aurora.

Funding: This research was supported by a grant from the Colorado Health Foundation (CHF-3848).

Conflict of interest: none declared.

Corresponding author: Melinda M. Davis, PhD, Department of Family Medicine, Oregon Health \& Science University, Mail Code FM, 3181 SW Sam Jackson Park Rd., Portland, OR 97239 (E-mail: davismel@ohsu.edu). havioral health services as a way to improve the quality of patient care. Terminology for and evidencebased models of integrated care are still emerging. ${ }^{3,5}$ Given the current emergence of language in this field, in this article we use the following definitions modified from Peek ${ }^{5}$ and Butler and colleagues. ${ }^{3}$ Behavioral bealth care is a broad term used to encompass care for patients around mental health and substance use conditions, health behavior change, life stressors and crises, as well as stress-related physical symptoms. Integrated care is care rendered by a practice team of primary care and behavioral health providers, working together with patients and families and using a systematic and cost-effective approach to provide patient-centered care. This care may address diverse behavioral health needs. Models of integrated care may vary on numerous levels, including but not limited to characteristics of the care team, spatial arrangements, type of collaboration, and protocols for patient detection, treatment, and follow-up. Com- 
mon integrated care models include collaborative depression care, primary care behavioral health, and co-located mental health and primary care services.

Evidence from randomized controlled trials (RCTs) demonstrates that integrated care improves process of care $e^{3,6-9}$ and clinical outcomes for patients with common medical and behavioral conditions (eg, diabetes, depression, anxiety), $3,6,8,10-16$ including patients' overall quality of life. , $^{3,6,8,11,12,17}$ Although RCTs suggest benefits to integrating patients and health care systems, ${ }^{17,18}$ widespread adoption has not occurred.

Several integration strategies, such as co-located services, shared medical records, and proactive referrals, have demonstrated effectiveness in RCTs. ${ }^{3}$ However, few studies describe the factors influencing implementation in real-world primary care practices. $^{4,19}$ Katon and colleagues ${ }^{20}$ note that even though collaborative depression care, one model of integrated care, has robust support in research, "it can be a great deal more challenging to implement such programs in the real world than to conduct the research to establish the evidence base for such programs." This is because approaches used in clinical efficacy studies may not be generalizable to other settings because of differences in staffing, leadership models, diverse patient care needs, or workflow processes. Many integration RCTs focus on single disease states or are conducted in large health care systems that have different reimbursement structures. ${ }^{3}$ Few real-world sites have the support of research personnel or financing for new positions that was available to the published studies. In addition, clinical trials tend to be randomized at the patient level rather than at the practice level and are focused on testing specific models of care (eg, collaborative depression care) rather than practice-level implementation of evidence-based integrated care strategies. ${ }^{3}$ Tailoring models so they are feasible and sustainable given local contexts and providing support during implementation may be necessary for integration to occur outside of RCTs. ${ }^{20-23}$

Research exploring how to implement and sustain integrated care in diverse primary care practices and behavioral health settings is needed. .,19,24 $^{2}$ Recent regional and national policy changes aimed at achieving a triple aim (ie, improved quality and experience of care while controlling costs), establishing patient-centered medical homes, and devel- oping accountable care organizations have led practices across the United States to tackle integration. ${ }^{3,23}$ We present early findings from 11 practices working to integrate care through the Advancing Care Together (ACT) program. ${ }^{25}$ ACT innovators are using multiple evidence-based strategies to integrate primary care and behavioral health. Our findings focus on 3 areas where all sites have encountered challenges in their early efforts: workflow/ access to care, leadership/culture change, and tracking/using data.

\section{The ACT Program}

ACT is a 4-year program of The Colorado Health Foundation (TCHF) that is designed to test strategies to integrate care for patients in real-world primary care and behavioral health settings. ${ }^{25}$ The fundamental goal of ACT is to change practices by addressing whole-person versus silo-like care. TCHF identified the Department of Family Medicine at the University of Colorado, Denver, as the Program Office for ACT. The Program Office selected a steering committee composed of Colorado and national leaders in primary care and behavioral health that helped conceptualize ACT and now provide guidance and direction for ongoing activities. TCHF also funded an evaluation of ACT.

ACT invited applicants to implement evidenced-based strategies to integrate care in their respective settings. ACT innovators received $\$ 50,000$ annually for a 3 -year period (totalling $\$ 150,000$ ). Funds were intended to offset costs of participating in the ACT evaluation and other grant-related responsibilities but were purposefully limited to avoid financing substantial aspects of the innovation. This was done to increase the likelihood that changes would be sustained beyond grant funding.

Primary care practices and behavioral health organizations in Colorado submitted applications for ACT in April 2011. ${ }^{25}$ Proposals were excluded if the setting was so unique that findings were unlikely to be replicable, if the proposed innovations did not use evidenced-based strategies, or if it focused on a single disease or condition. The ACT Steering Committee conducted an initial review of applications in May 2011. The evaluation team and Program Office conducted an evaluability assessment ${ }^{26}$ between June and August 2011 to gather additional information about semifinalists. 
In July 2011, the Steering Committee purposefully selected 11 applicants to create a diverse portfolio of evidence-based integration strategies, henceforth referred to as "ACT innovators" or "innovators." Funding started September 1, 2011. The Program Office developed learning community activities for ACT innovators, including bi-annual meetings, webinars, and personalized technical assistance.

\section{Methods}

We designed a mixed-method evaluation to observe the implementation of integrated care among the ACT innovators and to compare the reach ${ }^{27}$ and effectiveness of these efforts. The Oregon Health \& Science University and the University of
Texas Health Science Center at Houston institutional review boards approved the study protocol.

\section{Data Collection}

A multidisciplinary research team (epidemiologist, primary care clinician, clinical psychologist, experimental psychologist, communication scientist) with expertise in practice transformation, mixed methods, and integrated care is conducting the ACT evaluation. Table 1 describes the data informing this article, including documents (eg, grant application, semiannual reports); practice surveys; field notes from observation visits and ACT meetings; semistructured interviews; and online diaries. Online diaries are a web-based platform where participants can record real-time implementation experiences. ${ }^{28}$

Table 1. Data Sources and Measures for the Advancing Care Together (ACT) Evaluation

\begin{tabular}{|c|c|c|c|}
\hline Research Questions & $\begin{array}{l}\text { Primary Data } \\
\text { Type }\end{array}$ & Description of Data & Data Collection Process \\
\hline $\begin{array}{l}\text { How do the ACT practices } \\
\text { make the changes } \\
\text { required to integrate } \\
\text { care for patients? }\end{array}$ & Documents & $\begin{array}{l}\text { Documents include grant applications, } \\
\text { reports to TCHF, E-mail } \\
\text { communications, innovator } \\
\text { presentations, and documents from } \\
\text { innovators (scheduling templates, } \\
\text { educational materials). }\end{array}$ & $\begin{array}{l}\text { Documents are collected throughout } \\
\text { the study period and during } \\
\text { observation visits. The Program } \\
\text { Office and grantees share } \\
\text { documents freely. }\end{array}$ \\
\hline \multirow[t]{4}{*}{$\begin{array}{l}\text { What factors enable and } \\
\text { impede efforts to } \\
\text { integrate care for } \\
\text { patients, with particular } \\
\text { attention to teamwork, } \\
\text { information exchange, } \\
\text { and shared decision } \\
\text { making? }\end{array}$} & Online diaries & $\begin{array}{l}\text { Members from each innovation team } \\
\text { report their implementations } \\
\text { experiences biweekly via an online } \\
\text { journal that is shared with other } \\
\text { members of their team and the } \\
\text { evaluation team. }\end{array}$ & $\begin{array}{l}\text { The evaluation team identified } 5 \text { to } \\
7 \text { people on each grantee team, } \\
\text { including practice members, to } \\
\text { post diary entries. Each team has } \\
\text { a private online diary room. Diary } \\
\text { keepers were asked to post every } \\
2 \text { weeks. Evaluators interact with } \\
\text { diary keepers to encourage } \\
\text { posting. }\end{array}$ \\
\hline & Observation visits & $\begin{array}{l}\text { Two-day visits with each innovation } \\
\text { site to observe care delivery. }\end{array}$ & $\begin{array}{l}\text { During visits, } 2 \text { to } 3 \text { evaluators } \\
\text { observed the care delivery process } \\
\text { by shadowing clinicians, clinical } \\
\text { support staff, and nonclinical } \\
\text { support staff. This included } \\
\text { observing huddles and other team } \\
\text { meetings. }\end{array}$ \\
\hline & Interviews & $\begin{array}{l}\text { Informal and semistructured } \\
\text { interviews are conducted with the } \\
\text { innovators, clinic members, and } \\
\text { eventually patients. }\end{array}$ & $\begin{array}{l}\text { The evaluation team has informal } \\
\text { discussions with innovators during } \\
\text { meetings convened by the } \\
\text { Program Office. In addition, we } \\
\text { conduct semistructured interviews } \\
\text { with } 8 \text { to } 10 \text { practice members } \\
\text { during observation visits. }\end{array}$ \\
\hline & Survey & $\begin{array}{l}\text { A survey was completed by each } \\
\text { innovation team to collect } \\
\text { information about each } \\
\text { organization (eg, ownership, staffing } \\
\text { patterns, turnover, panel } \\
\text { characteristics). }\end{array}$ & $\begin{array}{l}\text { The evaluation team distributed } \\
\text { surveys to one person at each } \\
\text { ACT innovation site who worked } \\
\text { with members of the practice to } \\
\text { complete the information. } \\
\text { Information was returned to us } \\
\text { and data reviewed. Questions } \\
\text { were clarified with teams as } \\
\text { needed. }\end{array}$ \\
\hline
\end{tabular}

TCHF, The Colorado Health Foundation. 
Figure 1. Relationships at the organizational level.

\begin{tabular}{|c|c|c|c|c|}
\hline $\begin{array}{l}\text { Isolation / Mutual } \\
\text { Awareness }\end{array}$ & Cooperation & Collaboration & Partnership & $\begin{array}{l}\text { Merger / Single } \\
\text { Organization }\end{array}$ \\
\hline $\begin{array}{l}\text { Isolation: } \\
\text { Entities working } \\
\text { completely } \\
\text { separately } \\
\text { Mutual } \\
\text { Awareness: } \\
\text { Agencies are } \\
\text { informed about } \\
\text { each other and } \\
\text { each others' } \\
\text { activities }\end{array}$ & $\begin{array}{l}\text { Denotes some } \\
\text { sharing of } \\
\text { resources, such } \\
\text { as space, data, } \\
\text { or personnel }\end{array}$ & $\begin{array}{l}\text { Involves joint } \\
\text { planning and } \\
\text { execution, with } \\
\text { both entities } \\
\text { working } \\
\text { together to } \\
\text { coordinate at } \\
\text { multiple points } \\
\text { to carry out a } \\
\text { combined effort }\end{array}$ & $\begin{array}{l}\text { Implies } \\
\text { programmatic } \\
\text { integration, with } \\
\text { two entities } \\
\text { working so } \\
\text { closely together } \\
\text { that there is no } \\
\text { separation from } \\
\text { the end user's } \\
\text { perspective; } \\
\text { there are, in } \\
\text { fact, two } \\
\text { parties, but } \\
\text { their degree of } \\
\text { integration is so } \\
\text { great that the } \\
\text { effect is nearly } \\
\text { seamless }\end{array}$ & $\begin{array}{l}\text { Merger: One } \\
\text { combined entity } \\
\text { replacing the } \\
\text { formerly } \\
\text { separate } \\
\text { entities } \\
\text { Single } \\
\text { Organization: } \\
\text { One } \\
\text { organization to } \\
\text { start }\end{array}$ \\
\hline
\end{tabular}

\section{Data Management}

Paper surveys were manually entered into Excel (Microsoft Corp, Redmond, WA). Members of the evaluation team took notes during observation visits and used these to prepare field notes, typically within 24 hours. Interviews were audio-recorded and professionally transcribed. All data, including online diary data, were de-identified. Qualitative data were entered into Atlas.ti (ATLAS.ti Scientific Software Development $\mathrm{GmbH}$, Berlin, Germany) and survey data was entered into SAS software (SAS Inc., Cary, NC) for analysis.

\section{Data Analysis}

We used a grounded theory approach and analyzed data as we collected it. ${ }^{29}$ This involved reading and reviewing qualitative data and discussing as a team the larger patterns and lessons emerging across the 11 innovations. We did this in 3 immersion-crystallization cycles. ${ }^{30}$ First, we immersed ourselves in the data, meeting weekly to analyze each case (ie, individual practices/innovator teams). Through this process, key findings "crystallized" and were coded. In a second immersion-crystallization cycle, we analyzed how emerging findings manifest across innovations. In addition to identifying the importance of workflow and leadership, we found that innovators were at different developmental stages. To capture this distinction more formally and to refine our study findings further, we conducted a third immersion-crystallization cycle to characterize the organizational and interpersonal relationships present in each innovation using existing typologies (Figures 1 and 2). ${ }^{31,32}$

\section{Figure 2. Relationships at the interpersonal level.}

\begin{tabular}{|l|}
\hline Referral-triggered \\
periodic exchange \\
\hline Information \\
exchanged \\
periodically with \\
minimally shared \\
care plans or \\
workflows \\
\hline
\end{tabular}

\begin{tabular}{|l}
\hline \multicolumn{1}{c}{$\begin{array}{c}\text { Regular } \\
\text { communication / } \\
\text { coordination }\end{array}$} \\
Regular \\
communication and \\
coordination, usually \\
via separate \\
systems and \\
workflows, but with \\
care plans \\
coordination to a \\
significant extent \\
\end{tabular}

\begin{tabular}{|l}
\hline $\begin{array}{c}\text { Full collaboration / } \\
\text { integration }\end{array}$ \\
Fully shared \\
treatment plans and \\
documentation, \\
regular \\
communication \\
facilitated by a care \\
coordinator and/or \\
clinical workflows \\
that ensure effective \\
communication and \\
coordination of care
\end{tabular}




\section{Results}

\section{Description of ACT Innovators}

ACT is funding 9 primary care practices and 2 behavioral health agencies. Practices are located in rural and urban settings, represent private and public ownership models, and serve diverse payer and patient mixes (Table 2). Before ACT 4 primary care practices employed behavioral health providers; neither behavioral health agency employed medical clinicians.

Table 3 describes the 11 innovations, which are 15 months into implementation. Five innovators are integrating behavioral health into primary care, 2 are integrating primary care into behavioral health, and 4 are refining existing integration efforts. ACT innovators are past start-up (eg, selecting the integration model, developing initial staffing plans, hiring new professionals) and are actively implementing evidence-based strategies, including systematic screening, co-located care, and shared electronic health records (EHRs) (Table 3). ${ }^{3,33-41}$ Innovators have achieved integration of behavioral health and primary care services in 3 ways: by building partnerships with local and regional organizations, by directly hiring new personnel, or by expanding hours for existing behavioral health and medical clinicians.

Many innovators are developing or expanding partnerships with external organizations to implement integrated care. As portrayed in Figure 1, relationships between partnering organizations vary along a spectrum, from isolation (working separately) to merger (one combined entity replaces formerly separate entities). ${ }^{32}$ Innovators move across this spectrum as organizations work together over time. At the time of our analysis, 4 of the ACT innovators (IDs 4, 6, 9, 11) involved a single organization with behavioral health and medical departments. Two innovators (IDs 1 and 3) are at the "cooperation stage," with organizations sharing physical space, data, and some ancillary staff. Two (IDs 2 and 10) are transitioning from the "cooperation" to the "collaboration stage" as organizations begin to jointly plan and execute goals. Three innovators (IDs $5,7,8$ ) are in the early stages of "collaboration" (these partnering organizations have joint goals) and are working on various strategies (eg, shared schedules, shared care plans) to deliver integrated care.
At the level of interpersonal relationships, innovators employ multiple strategies to enable integrated care (Figure 2; Table 3). ${ }^{5}$ Six ACT Innovators (IDs 1, 2, 3, 5, 6, 9) predominantly use a "referral-triggered" strategy, where information is shared on an as-needed basis, with minimally shared care plans and workflows. Four innovators (IDs 4, 7, 8, 10) use "regular communication/coordination" as their dominant strategy but revert to a referral-triggered method when behavioral health providers are not available. In the regular communication/coordination approach providers regularly communicate with each other about patients and coordinate care plans, even if they are using separate EHRs or have separate workflows. For example, in one clinic, behavioral health providers route treatment notes to medical clinicians who review then reinforce behavioral health goals during medical encounters. One innovator (ID 11) is at "full collaboration/integration" based on initial organizational structure.

\section{Early Challenges to Integration}

ACT innovators are facing challenges in their early integration efforts in 3 areas: workflow and access, leadership and culture change, and tracking and using data. These challenges are manifesting across all sites, irrespective of care setting or integration focus. The next section uses the case narrative of one innovator (ID 7) to illustrate these challenges.

\section{Key Challenges and Early Solutions to Integration: ACT Innovator 7 Case Narrative}

Primary care practice A is located in Colorado suburb and has been exploring opportunities to integrate behavioral health for several years. Established more than 50 years ago, the practice is currently owned by 2 physicians and employs 4 medical clinicians (physician, physician assistant, nurse practitioner, and chiropractor), an office manager, and 17 clinical and nonclinical support staff. The practice refers patients to a community mental health center (CMHC) and has rented space to a local psychologist for 2 evenings a week for the past few years. These arrangements provide access to behavioral health services for patients with the right types of insurance and diagnoses. However, brief intake screens and the lived experience at the practice indicate that many patients have mild to moderate behavioral health needs that are unmet. 


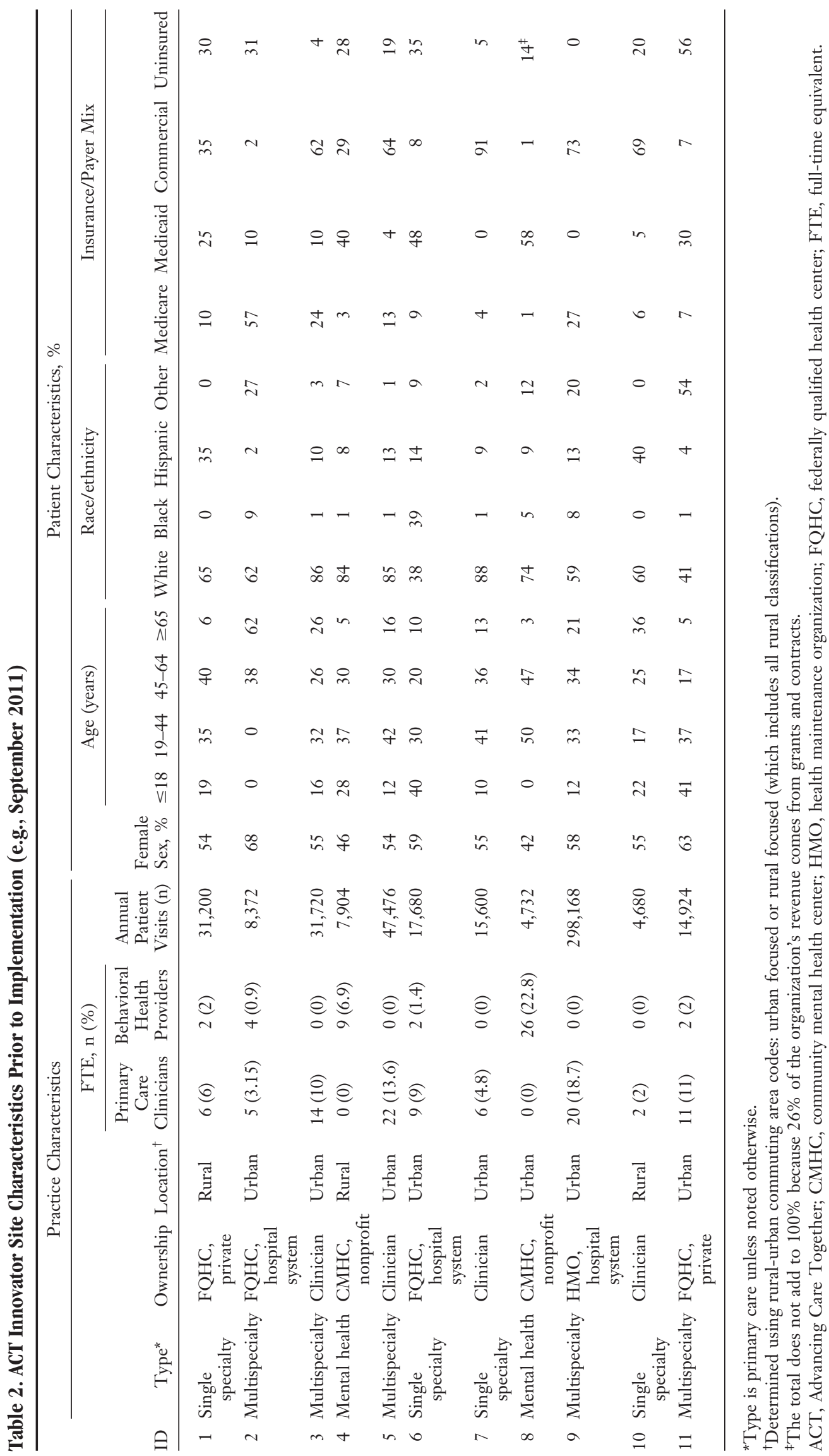




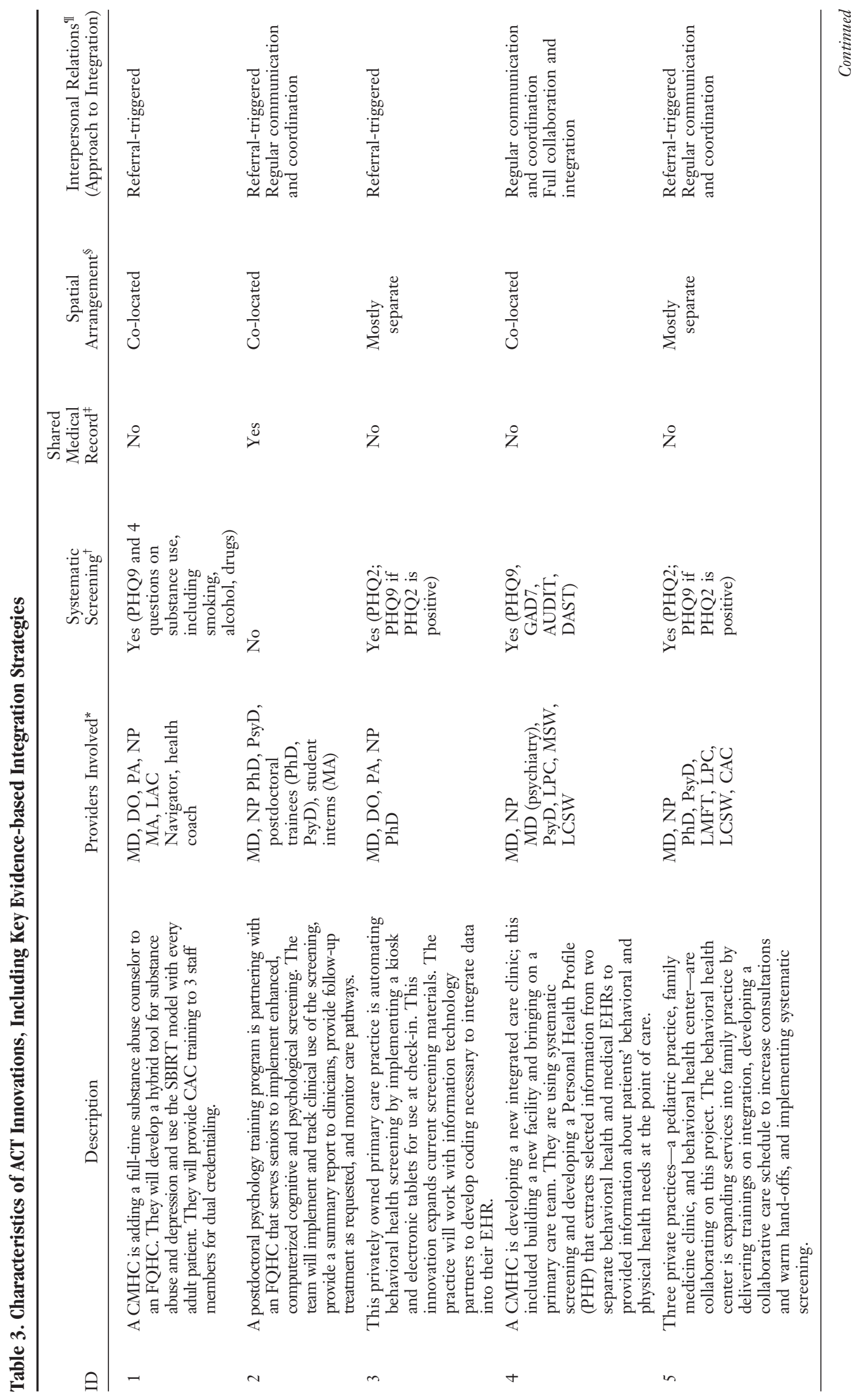




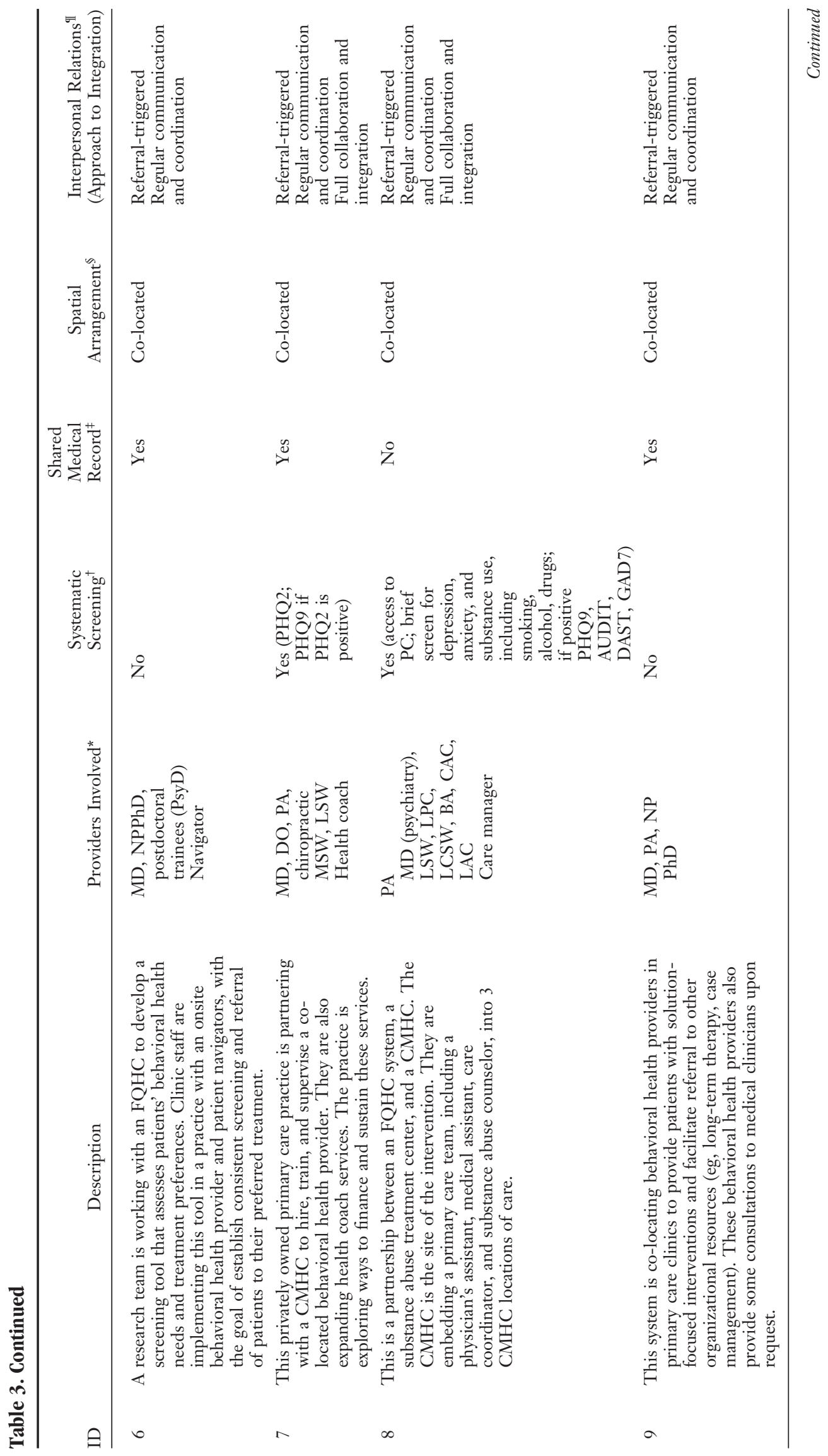




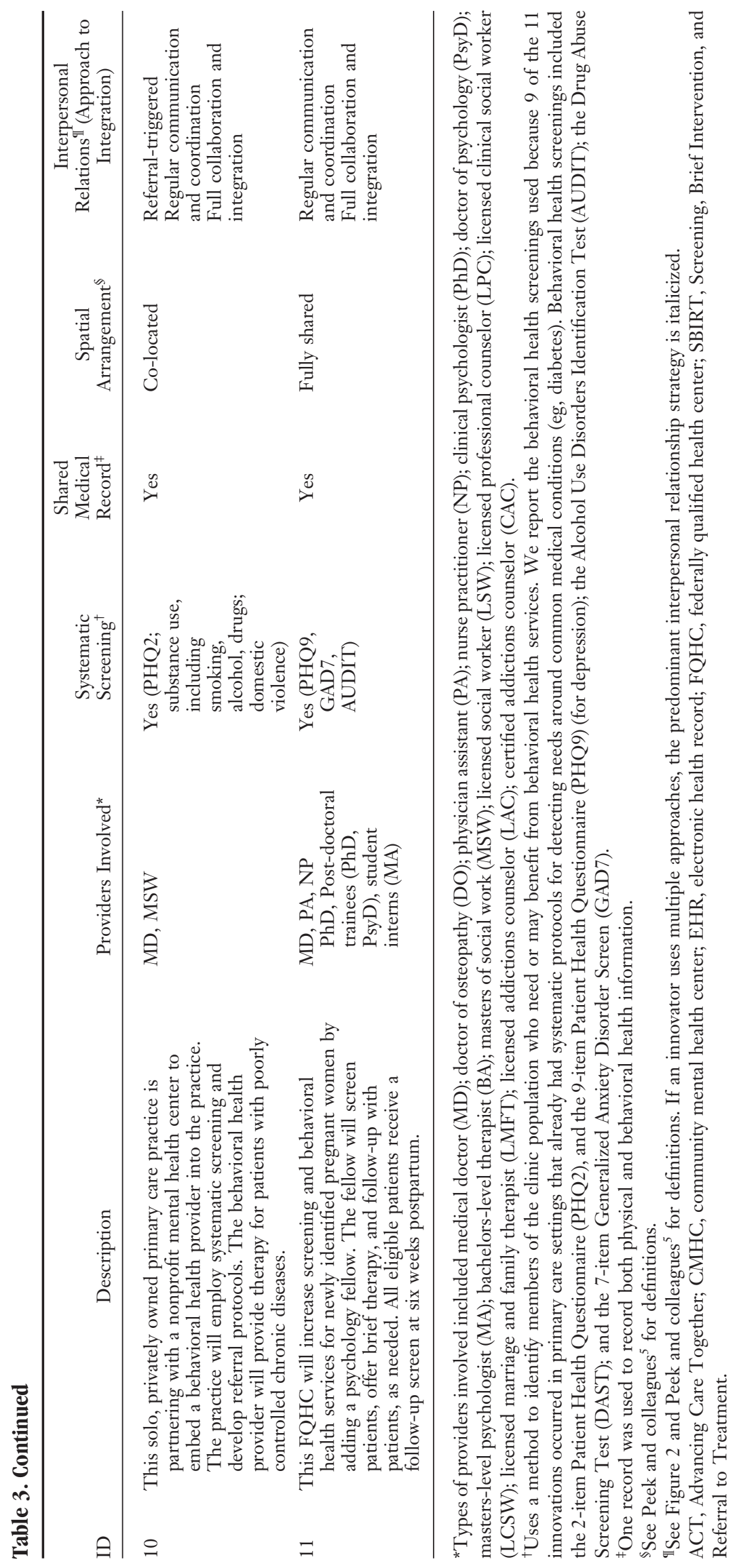


The medical clinicians want a behavioral health provider to help treat patients in real time.

From a financial perspective, the practice is not able to hire a full-time behavioral health provider. When practice A learned of ACT, they saw it as an opportunity to integrate a behavioral health provider, expand their health coaching program, and evaluate the financial sustainability of this model using a hypothetical fee of $\$ 6$ per member per year that would be paid by patients. Practice A had worked with the local $\mathrm{CMHC}$ on a few previous projects, but the timing had never been right to foster integrated care. When ACT was announced the practice knew they did not want to collaborate with an individual and so the medical director and clinic manager approached the CMHC to see whether their behavioral health services could be expanded to the practice. After several months, both organizations agreed on a financial strategy to place a behavioral health provider in practice A 2 days a week. The CMHC was simultaneously building integrated partnerships with 5 other primary care clinics.

Hiring the part-time behavioral health provider was a joint effort by the primary care clinic and CMHC. It took several months to identify a candidate with the right clinical skills who was willing to work for the hours and pay available. The behavioral health provider participated in a weeklong orientation at the CMHC and was introduced to the practice. This person participated in monthly primary care clinic staff meetings and received office space in the practice in which to see patients. A team of clinicians and staff developed protocols for how to access the behavioral health provider. This included a behavioral health schedule with 40-minute therapy appointments interspersed with 30-minute blocks for open consultation. This schedule was shifted several times in response to provider and staff feedback. When the behavioral health provider was not on site, the practice developed alternate strategies (including an introductory pamphlet and paper-based referral form) to support patient connections. However, it was challenging to make warm hand-offs (eg, bringing another professional into the visit to introduce this person to the patient and transition care) and hallway consultations a routine part of care. This was because of the behavioral health provider's limited hours, as well as challenges integrating primary care members and the behavioral health provider's personalities and professional identities. Shortly after her placement, the behavioral health provider left for a full-time position in another setting.

After several months, the practice and CMHC partners hired another behavioral health provider. However, medical clinicians built up a backlog of behavioral health referrals during the interim, and when the new behavioral health provider started, her schedule was booked solid with previously referred patients. This limited time for warm-hand offs and consultations. The new behavioral health provider found it difficult to balance the competing demands of the position (eg, providing therapy and brief consults, following up on referrals, preparing chart notes, participating in staff and leadership meetings). Building rapport with practice members was also a challenge. She left the position after a few weeks. The practice and CMHC recently hired their third behavioral health professional.

As the CMHC expanded their focus to place behavioral health providers in primary care settings, they created a position to help manage their integrated care initiatives. This CMHC employee supervises the integrated behavioral health providers at 6 partnering primary care practices (including primary care practice A), builds rapport with primary care practice members, and serves as a liaison between the CMHC and the integrated practices. Despite everyone's best efforts, practice A is frustrated at the slower pace of decision making at the CMHC, which is a large organization in which decision making takes longer than it does in smaller, independent organizations. Both partners recognize that it would be ideal to have the next behavioral health provider onsite full time, have time available for consults and warm hand-offs, and actively involve this person in developing the emerging model of integrated care. We believe that both parties also are recognizing that successful integration of the behavioral health provider is likely to require a culture and identify shift by both the primary care members and the behavioral health professional.

\section{Workflow and Access to Care}

ACT innovators are developing workflows to ensure consistent access to integrated care. However, when new medical or behavioral health professionals are added to a practice, they are typically hired (or contracted) as part-time employees. Part-time 
schedules and other factors (eg, vacation, illness, continuing education, semester transitions in training programs) pose barriers to access. Even when medical and behavioral health providers are present simultaneously, demands of patient care sometimes render professionals inaccessible, making it difficult to implement consistent workflows.

Innovators are addressing these challenges by expanding hours for part-time providers, developing schedules that intersperse short counseling sessions with time for warm hand-offs and hallway consultations, and creating rules for interruption (eg, practices establish policies allowing medical or behavioral health providers to be interrupted during an encounter if another patient is in crisis). Innovators also are building "contingency" workflows to support asynchronous patient hand-offs. For instance, at site 7 , if the behavioral health provider is not available medical clinicians can distribute a brochure to the patient describing behavioral health services and send a message via the EHR to the behavioral health provider to initiate a phone consultation. Innovators also are developing scripts to inform patients about new integrated care resources, especially behavioral health services.

\section{Leadership and Culture Change}

Integration is a fundamental change in practice culture. New collaborations are difficult to foster among professional equals, especially when one professional joins an organization led by the another group of professionals (eg, a clinician/medical assistant team embedded in behavioral health setting; a psychologist embedded in a primary care practice). Differences in opinion emerge, and we observed struggles around who controls patientprovider relationships, how perspectives on patient needs and diagnoses are managed, what is an appropriate pace of care delivery (eg, shortening behavioral health sessions), and how to complete and share encounter notes. Staff turnover is common. Finding the "right" people for the integrated care team is necessary but insufficient for solving these problems. In behavioral health training programs, power struggles are minimized because students are in a learning/supportive role and are acculturated into the primary care model. ${ }^{42,43}$

Leadership influences how professional interactions unfold and whether teams are able to innovate, "fail," and try again when implementing integration. When interventions involve multiple organizations, leadership from each setting must inform the integrated care approach. New supervisory positions are emerging to support integrated staff. These individuals bridge unique organizations, frequently facilitating and mediating interpersonal relationships and organizational objectives. In addition, leadership is critical to engaging on-the-ground professionals in decisions, such as changing physical layout, relocating providers to shared office spaces, and creating collaborative meeting times (eg, team huddles, time during staff meetings) that facilitate new ways of working together and foster camaraderie and culture change.

\section{Tracking Patients and Using Data}

Before ACT, few innovators systematically screened patients for behavioral health needs. Early during implementation, innovators selected evidence-based, pragmatic screens for common physical (eg, body mass index, blood pressure) or behavioral health (eg, depression, anxiety, substance use) conditions. Many innovators developed screening protocols and are now systematically collecting patient-level data (Table 3). Innovators are learning how to use these data to: (1) monitor patient improvement and escalate treatment as needed, (2) manage care for a population of patients (eg, those with uncontrolled diabetes) and reach out to patients where behavioral health patterns may present barriers to wellness; and (3) monitor practice progress with regard to care quality.

Although innovators recognize the value of using data to support care for individual patients, few sites have EHRs designed to capture both physical and behavioral health information or to share this information between clinical disciplines. Several are navigating 2 EHR systems to access and record patient data. In addition, data on behavioral health processes (eg, referral to psychiatrist) and outcome indicators (eg, depression screening scores on the 9-item Patient Health Questionnaire) are not routinely captured in discrete fields, making it difficult to run population-level queries to evaluate practice change. Moreover, few sites have the staffing infrastructure to run or review these queries.

Innovators are creating workarounds to record and/or access relevant patient data because health information technology is inadequate for integrated care. Two innovators (IDs 3 and 4) are automating screening with a tablet that syncs with 
the EHR in real time. Others are building registries of patients who screen positive and maintaining them through manual data entry. Some are creating EHR-based documentation templates and developing new data query scripts.

\section{Discussion}

Innovators are using ACT as an opportunity to "start where they are" and build an infrastructure for integrated care that can be clinically, operationally, and financially sustained beyond the funding period. In 15 months, innovators have made progress in both organizational and interpersonal relationships as they integrate care. We highlight 3 challenges the innovators are addressing: developing workflows and managing access to new health providers, fostering changes in culture necessary for integration, and improving data tracking and use to monitor quality of care at the patient and practice levels. Our findings provide a window into the complicated and difficult process of transforming primary care and behavioral health settings into integrated care facilities.

Although RCTs demonstrate that integration decreases fragmentation and improves comprehensive care delivery and patient outcomes, ${ }^{3,44,45}$ there is little guidance on how to translate findings from these well-supported research studies into realworld practices. ${ }^{46}$ The Agency for Healthcare Research and Quality's 2008 review of evidence on the efficacy of integrated care indicates that numerous organizational and financial barriers to successful integration persist; few clinical trials, however, describe barriers to implementation or the strategies employed to overcome them. ${ }^{3}$ For instance, one trial identified limited time as a barrier to implementing a 2-step screening process for depression in the primary care setting. To address this, the research team hired additional staff to screen patients, ${ }^{3,47}$ a solution not often feasible in real-world settings. In addition, facilitative leadership and developing interprofessional relationships are critical to enabling integration. Yet most trials do not report or offer strategies to address the leadership and relationship challenges that manifest across innovators sites and likely across all practices attempting integration., ${ }^{3,48}$ By collecting in-depth data during implementation, we provide rich insights into what works and what does not as practices implement evidence-based integration strategies in real-world settings.
Various strategies for integration continue to be developed. ${ }^{45,49}$ Our experience and that of others suggests that during implementation integrated care strategies must be tailored to the resources available in the local practice and community settings. ${ }^{23}$ In addition, integration requires improving teamwork and collaboration, ${ }^{14,50-52}$ developing clinical skills, ${ }^{14,51-53}$ supporting clinician engagement, and improving the exchange of health information. ${ }^{19}$ Exploring how practices develop the skills and tools needed to integrate care and tailor integration strategies outside of RCTs is an important advance for the field. ${ }^{24,54}$

The ACT portfolio provides rich data on a range of strategies real-world practices use to integrate care. Innovators demonstrate that integration is not accomplished by simply adding screening protocols and new professionals to a practice. Although being physically present may create opportunities to provide whole-person care, we observed that integration requires fundamental changes in how health care professionals view their roles. It requires developing organizational and interpersonal relationships. Even within an organization, implementing integrated care may require subgroups within the organization, who may be working together for the first time, to develop new ways of relating. Some innovators have stated that, "identity change is fine, as long as it is not mine." Leadership plays a critical role in helping people make the changes to culture and identity that are necessary for integrating care. It is important that leaders change, too, and external support may be needed to help practice members overcome early challenges.

Virtually every study of integrated care targets populations of patients with a single disease. ${ }^{3}$ In contrast, ACT innovators provide integrated care to the full spectrum of patients who present in their practices and treat patients with a range of conditions-from simple to complex, behavioral to physical-within the available infrastructure. To accomplish this, innovators design flexible workflows and test, evaluate, and refine work processes through short, naturally occurring change cycles. Yet innovators often do not have the data needed to know if these short cycle changes are working. ${ }^{55,56}$ We anticipate that this data problem is not insurmountable. It requires EHR systems designed for integrated practices, and few exist in today's marketplace. In addition, practices must invest capital to purchase better EHR systems and, once they are 
available, to train personnel to use these systems for data extraction and quality improvement.

The mixed-methods case comparison design we use in the ACT evaluation allows us to observe nuances across diverse practices and illustrate the dynamic ways practices integrate evidenced-based integration strategies. These early findings would be obscured in traditional study designs. In ACT, we couple real-time qualitative data collection (through diaries, interviews, observation visits) to understand how implementation occurs and quantitative methods (through data tracking) to assess impact. Rigorous comparative case studies that employ a repeated time series design to assess outcomes may be better aligned with the goal of informing subsequent dissemination or scale-up efforts than RCTs. ${ }^{4,57-60}$

There are limitations of this study. First, ACT innovators are located in Colorado, a state ripe for integrated care innovation because of the support and advocacy of local foundations as well as academic and political leadership. However, the challenges emerging as innovators work to sustain integration are likely to be common across many practices and states. Second, we characterize innovators' organizational and interpersonal relationships at a single point in time; this is our team's assessment based on available data. Innovators are moving, sometimes rather rapidly, along these spectrums. Finally, our article describes findings from early during implementation. It is too early to know whether innovations will improve patient outcomes and to assess the financial implications of these changes. However, our early findings shed light on how real-world practices experience the initial stages of implementing evidence-based integrated care.

The IOM identifies the need for research to understand how to implement and sustain evidence-based interventions for integrated care in everyday practice. ${ }^{4,61}$ ACT is addressing this need. As the health policy landscape continues to change (eg, accountable care organizations, patient-centered medical homes) and incentives align to finance integrated care, more practices in the United States will embrace this approach. ${ }^{23}$ Our findings demonstrate that integration requires improving workflow and access, using leadership to support culture change, and developing the capacity for tracking data to inform patient care and continuous quality improvement. We need to identify ways to assist practices that are integrating care in addressing these implementation challenges and continue to study these efforts so we can learn from frontline innovators how best to take evidence-based integration to scale.

The authors thank the 11 practices (and their collaborators) participating in the ACT program. We appreciate the assistance of Rose Gunn, MA, and Frank V. DeGruy III, MD, MSFM, in the preparation of this manuscript.

\section{References}

1. Donaldson MF, Yordy KD, Lohr KN, Vanselow NA, eds. Primary care: America's health in a new era. Washington, D.C.: The National Academies Press; 1996. Available from: http://www.nap.edu/ openbook.php?record_id $=5152$ \&page $=$ R1. Accessed July 19, 2013.

2. Croghan TW, Brown JD. Integrating mental health treatment into the patient-centered medical home. Rockville, MD: Agency for Healthcare Research and Quality; 2010. Available from: http://www.pcpcc.org/ resource/integrating-mental-health-treatment-patientcentered-medical-home. Accessed July 19, 2013.

3. Butler M, Kane RL, McAlpine D, et al. Integration of mental health/substance abuse and primary care. Evidence report/technology assessment no. 173. Rockville, MD: Agency for Healthcare Research and Quality; 2008. Available from: http://www.ahrq.gov/research/ findings/evidence-based-reports/mhsapc-evidencereport.pdf. Accessed July 19, 2013.

4. Institute of Medicine. Improving the quality of health care for mental and substance-use conditions: Quality Chasm Series. Washington, D.C.: The National Academies Press; 2006. Available from: http://www.iom.edu/ Reports/2005/Improving-the-Quality-of-Health-Carefor-Mental-and-Substance-Use-Conditions-QualityChasm-Series.aspx. Accessed July 19, 2013.

5. Peek CJ; the National Integration Academy Council. Lexicon for behavioral health and primary care integration: concepts and definitions developed by expert consensus. Rockville, MD: Agency for Healthcare Research and Quality; 2013. Available from: http:// integrationacademy.ahrq.gov/sites/default/files/Lexicon. pdf. Accessed July 19, 2013.

6. Hunkeler EM, Katon W, Tang L, et al. Long term outcomes from the IMPACT randomised trial for depressed elderly patients in primary care. BMJ 2006;332:259-63.

7. Katon W, Russo J, Von Korff M, et al. Long-term effects of a collaborative care intervention in persistently depressed primary care patients. J Gen Intern Med 2002;17:741-8. 
8. Hedrick SC, Chaney EF, Felker B, et al. Effectiveness of collaborative care depression treatment in Veterans' Affairs primary care. J Gen Intern Med 2003;18:9-16.

9. Katon WJ, Von Korff M, Lin EH, et al. The Pathways Study: a randomized trial of collaborative care in patients with diabetes and depression. Arch Gen Psychiatry 2004;61:1042-9.

10. Unutzer J, Katon W, Callahan CM, et al. Collaborative care management of late-life depression in the primary care setting: a randomized controlled trial. JAMA 2002;288:2836-45.

11. Roy-Byrne PP, Katon W, Cowley DS, Russo J. A randomized effectiveness trial of collaborative care for patients with panic disorder in primary care. Arch Gen Psychiatry 2001;58:869-76.

12. Rost K, Nutting P, Smith JL, Elliott CE, Dickinson M. Managing depression as a chronic disease: a randomised trial of ongoing treatment in primary care. BMJ 2002;325:934.

13. Gilbody S, Bower P, Fletcher J, Richards D, Sutton AJ. Collaborative care for depression: a cumulative meta-analysis and review of longer-term outcomes. Arch Intern Med 2006;166:2314-21.

14. Katon W, Von Korff M, Lin E, et al. Collaborative management to achieve treatment guidelines. Impact on depression in primary care. JAMA 1995;273: 1026-31.

15. Wells KB, Sherbourne C, Schoenbaum M, et al. Impact of disseminating quality improvement programs for depression in managed primary care: a randomized controlled trial. JAMA 2000;283: 212-20.

16. Roy-Byrne P, Craske MG, Sullivan G, et al. Delivery of evidence-based treatment for multiple anxiety disorders in primary care: a randomized controlled trial. JAMA 2010;303:1921-8.

17. Unutzer J, Katon WJ, Fan MY, et al. Long-term cost effects of collaborative care for late-life depression. Am J Manag Care 2008;14:95-100.

18. Katon W, Unutzer J, Fan MY, et al. Cost-effectiveness and net benefit of enhanced treatment of depression for older adults with diabetes and depression. Diabetes Care 2006;29:265-70.

19. Kathol RG, Butler M, McAlpine DD, Kane RL. Barriers to physical and mental condition integrated service delivery. Psychosom Med 2010:72:511-8.

20. Katon W, Unutzer J, Wells K, Jones L. Collaborative depression care: history, evolution and ways to enhance dissemination and sustainability. Gen Hosp Psychiatry 2010;32:456-64.

21. Unutzer J, Powers D, Katon W, Langston C. From establishing an evidence-based practice to implementation in real-world settings: IMPACT as a case study. Psychiatr Clin North Am 2005;28:1079-92.

22. Grypma L, Haverkamp R, Little S, Unutzer J. Taking an evidence-based model of depression care from research to practice: making lemonade out of depression. Gen Hosp Psychiatry 2006;28:101-7.

23. Levey S, Miller B, deGruy F III. Behavioral health integration: an essential element of populationbased healthcare redesign. Transl Behav Med 2012;2:364-71.

24. Miller BF, Kessler R, Peek CJ, Kallenberg GA, Mullican C. A national research agenda for research in collaborative care. Rockville, MD: Agency for Healthcare Research and Quality; 2011. Available from: http://www.ahrq.gov/research/findings/final-reports/ collaborativecare/. Accessed July 19, 2013.

25. University of Colorado Department of Family Medicine. Advancing Care Together [homepage]. Available from: http://www.advancingcaretogether.org/. Accessed January 3, 2013.

26. Leviton LC, Collins CB, Laird BL, Kratt PP. Teaching evaluation using evaluability assessment. Evaluation 1998;4:389-409.

27. Virginia Polytechnic Institiute and State University. Reach Effectiveness Adoption Implementation Maintenance (RE-AIM) [homepage]. Available from: http:// www.re-aim.org/. Accessed January 3, 2013.

28. Cohen DJ, Leviton LC, Isaacson N, Tallia AF, Crabtree BF. Online diaries for qualitative evaluation: gaining real-time insights. Am J Evaluation 2006;27:163-84.

29. Glaser BG, Strauss AL. The discovery of grounded theory: strategies for qualitative research. Chicago, IL: Aldine; 1967.

30. Borkan J. Immersion/crystallization. In: Crabtree BF, Miller WL, eds. Doing qualitative research. 2nd ed. Thousand Oaks, CA: Sage Publications; 1999: 179-94.

31. Blount A. Integrated primary care: organizing the evidence. Fam Syst Health 2003;21:121-33.

32. Institute of Medicine. Primary care and public health: exploring integration to improve population health. Washington, D.C.: National Academies Press; 2012. Available from: http://www.iom.edu/Reports/2012/ Primary-Care-and-Public-Health.aspx. Accessed July 19, 2013.

33. Bernstein E, Bernstein J, Feldman J, et al. An evidence based alcohol screening, brief intervention and referral to treatment (SBIRT) curriculum for emergency department (ED) providers improves skills and utilization. Subst Abus 2007;28:79-92.

34. Finkel SI. Cognitive screening in the primary care setting. The role of physicians at the first point of entry. Geriatrics 2003;58:43-4.

35. O'Connor EA, Whitlock EP, Beil TL, Gaynes BN. Screening for depression in adult patients in primary care settings: a systematic evidence review. Ann Intern Med 2009;151:793-803.

36. Screening for depression in adults: U.S. Preventive Services Task Force recommendation statement. Ann Intern Med 2009;151:784-92.

37. Druss BG, Bornemann TH. Improving health and health care for persons with serious mental illness: 
the window for US federal policy change. JAMA 2010;303:1972-3.

38. Druss BG, von Esenwein SA. Improving general medical care for persons with mental and addictive disorders: systematic review. Gen Hosp Psychiatry 2006;28:145-53.

39. Wales P. Solution-focused brief therapy in primary care. Nurs Times 1998;94:48-9.

40. Gjerdingen DK, Yawn BP. Postpartum depression screening: importance, methods, barriers, and recommendations for practice. J Am Board Fam Med 2007;20:280-8.

41. Breedlove G, Fryzelka D. Depression screening during pregnancy. J Midwifery Womens Health 2011; 56:18-25.

42. Blount A, DeGirolamo S, Mariani K. Training the collaborative care practitioners of the future. Fam Syst Health 2006;24:111-9.

43. Talen MR, Fraser JS, Cauley K. Training primary care psychologists: a model for predoctoral programs. Prof Psychology Res Pract 2005;36:136-43.

44. Dickinson WP, Miller BF. Comprehensiveness and continuity of care and the inseparability of mental and behavioral health from the patient-centered medical home. Fam Syst Health 2010;28:348-55.

45. Strosahl K, Robinson P. The primary care behavioral health model: applications to prevention, acute care and chronic condition management. In: Kessler R, Stafford D, eds. Collaborative medicine case studies: evidence in practice. New York: Springer; 2008: 85-96.

46. Robinson PJR, Jeff T. Behavioral consultation and primary care: a guide to integrating services. New York, NY: Springer Science and Business Media; 2007.

47. Rost K, Nutting PA, Smith J, Werner JJ. Designing and implementing a primary care intervention trial to improve the quality and outcome of care for major depression. Gen Hosp Psychiatry 2000;22:66-77.

48. Kilbourne AM, Schulberg HC, Post EP, Rollman BL, Belnap BH, Pincus HA. Translating evidencebased depression management services to community-based primary care practices. Milbank Q 2004;82: 631-59.
49. Collins C, Hewson DL, Munger R, Wade T. Evolving models of behavioral health integration in primary care. New York: Milbank Memorial Fund; 2010. Available from: http://www.milbank.org/uploads/documents/ 10430EvolvingCare/10430EvolvingCare.html. Accessed July 19, 2013.

50. Craven M, Bland R. Better practices in collaborative mental health care: an analysis of the evidence base. Can J Psychiatry 2006;51(Suppl 1):7S-72S.

51. Katon W, Robinson P, Von Korff M, et al. A multifaceted intervention to improve treatment of depression in primary care. Arch Gen Psychiatry 1996; 53:924-32.

52. Peek CJ, Baird MA, Coleman E. Primary care for patient complexity, not only disease. Fam Syst Health 2009;27:287-302.

53. Hunter CL, Goodie JL. Operational and clinical components for integrated-collaborative behavioral healthcare in the patient-centered medical home. Fam Syst Health 2010;28:308-21.

54. Katon W, Unutzer J. Collaborative care models for depression: time to move from evidence to practice. Arch Intern Med 2006;166:2304-6.

55. Cunningham PJ. Beyond parity: primary care physicians' perspectives on access to mental health care. Health Aff (Millwood) 2009;28:w490-501.

56. Massa I, Miller BF, Kessler R. Collaboration between NCQA patient-centered medical homes and specialty behavioral health and medical services. Trans Behav Med 2012;1-5.

57. Kessler R, Glasgow RE. A proposal to speed translation of healthcare research into practice: dramatic change is needed. Am J Prev Med 2011;40:637-44.

58. Speroff T, O'Connor GT. Study designs for PDSA quality improvement research. Qual Manag Health Care 2004;13:17-32.

59. Berwick DM. The science of improvement. JAMA 2008;299:1182-4.

60. Pawson R, Tilly N. Realistic evaluation. London: Sage Publications; 1997.

61. Institute of Medicine. Fostering rapid advances in healthcare: Learning from systems demonstrations. Washington, D.C.: National Academies Press; 2002. 